\title{
Noninvasive Investigation of Deep Vascular Pathologies of Exudative Macular Diseases by High Penetration Optical Coherence Angiography
}

Young-Joo Hong, ${ }^{1,4}$ Masahiro Miura, ${ }^{2,4}$ Shuichi Makita, ${ }^{1,4}$ Myeong-Jin Ju, ${ }^{3}$ Byeong Ha Lee, ${ }^{3}$ Takuya Iwasaki, ${ }^{2}$ Yoshiaki Yasuno ${ }^{1,4}$

${ }^{1}$ Computational Optics Group, University of Tsukuba, Tsukuba, Ibaraki, Japan

${ }^{2}$ Department of Ophthalmology, Ibaraki Medical Center, Tokyo Medical University, Ami, Ibaraki, Japan

${ }^{3}$ School of Information and Communications, Gwangju Institute of Science and Technology, Gwangju, South Korea

${ }^{4}$ Computational Optics and Ophthalmology Group, Tsukuba, Japan

Running Head

Vascular Pathologies of Exudative Macular Diseases with Optical Coherence Angiography

Corresponding author:

Yoshiaki Yasuno, Ph. D.

Computational Optics Groups in the University of Tsukuba

1-1-1 Tennoudai, Tsukuba, Ibaraki, 305-8571, Japan

Phone and Fax: +81-29-853-5217

E-mail: yasuno@optlab2.bk.tsukuba.ac.jp

Keywords: Non-invasive angiography, Choroid, Vasculature, Doppler, Optical coherence tomography Article type: Original Article 


\section{Abstract}

Purpose: A newly developed high-penetration Doppler optical coherence angiography (HP-OCA) with a $1-\mu \mathrm{m}$ probe beam for non-invasive investigation of vascular pathology of exudative macular diseases is introduced. A descriptive case series is presented to discuss the clinical utility of HP-OCA.

Methods: Eleven eyes of 10 subjects with exudative macular disease, including 2 eyes with myopic choroidal neovascularization (mCNV), 4 eyes with age-related macular degeneration (AMD) and 5 eyes with polypoidal choroidal vasculopathy (PCV), were investigated. Two Doppler scanning modes (bi-directional and high-sensitive) of HP-OCA were used for the investigation. HP-OCA provides depth resolved and en face angiograms and a structural OCT noninvasively. The HP-OCA images were compared with fluorescein angiography (FA), indocyanine green angiography (ICGA) and color fundus images.

Results: The abnormal vasculature patterns observed with high-sensitive HP-OCA presented high similarity to the mid-phase of ICGA. Several abnormal Doppler signals were observed in the en face high-sensitive HP-OCA and were co-located with FA leakage. This co-location was found in 1 eye with mCNV, 4 eyes with AMD, and 1 eye with PCV. Doppler tomogram of the bi-directional mode showed abnormal Doppler signals in 3 of 5 PCV cases beneath the pigment epithelium detachment. With the high-sensitive mode, Doppler signals were found beneath the elevated retinal pigment epithelium in all untreated cases.

Conclusions: HP-OCA revealed depth-resolved abnormal vasculatures in exudative macular diseases. The en face HP-OCA images showed high similarity with FA and ICGA images. These results suggest HP-OCA can be used for non-invasive and three-dimensional angiography in a clinical routine. 
Exudative macular diseases threaten vision ability of humans. Among them, pathological myopia and age-related macular degeneration (AMD) are representative causes of blindness worldwide ${ }^{1-5}$. These diseases frequently appear with choroidal neovascularization (CNV). Polypoidal choroidal vasculopathy (PCV) is one form of $\mathrm{CNV}$ and it is characterized by a branching vascular network (BVN) terminating in polypoidal lesions ${ }^{6,7}$. In Asian countries, PCV represents between $56 \%$ and $72 \%$ of all neovascular AMD patients ${ }^{1}$.

One of the most effective treatments of $\mathrm{CNV}$ is intravitreal injection of bevacizumab or ranibizumab. This treatment requires repeated injection with several rounds with 1- to 3-month periods, and fluorescence angiography (FA) and indocyanine green angiography (ICGA) images are required for the initial diagnosis. However, FA and ICGA imaging is sometimes restricted, because they are invasive methods requiring dye injection into a vein, which makes patients uncomfortable, and sometimes induces adverse reactions ${ }^{8,9}$. Hence, it is clinically important to establish a new modality that non-invasively reveals the vasculature including abnormal vasculature.

Optical coherence tomography (OCT) has been an innovative ophthalmic diagnostic modality that provides non-invasive optical biopsy imaging ${ }^{10}$. However, conventional OCT with an $840 \mathrm{~nm}$ probe beam can visualize only limited structures beneath the RPE. It is known that light with a wavelength of around $1050 \mathrm{~nm}$ is less scattered in the choroid and less absorbed in the RPE, and OCT with a $1050 \mathrm{~nm}$ probe beam, also referred to as high-penetration OCT (HP-OCT), has been demonstrated for high-penetration imaging of the posterior eye ${ }^{11}$. Visualization of sub-RPE morphologies in exudative macular disease by HP-OCT has also been demonstrated ${ }^{12,13}$.

In addition to structural imaging, Doppler OCT, a functional extension of OCT, has been demonstrated to image flow in living tissues ${ }^{14-20}$. Doppler OCT provides depth-resolved flow contrast by detecting the Doppler shift of a probe beam induced by the motion of scatterers in the sample. This technique and its variations are utilized both for quantitative flow investigation ${ }^{21-24}$ and detailed structural investigation of the vasculature ${ }^{25-29}$. The former is now possible with a commercially available OCT device, such as the RTView (Optovue Inc. CA). The latter, so called OCT angiography or optical coherence angiography (OCA), is still in a research stage, but has been successfully applied in the investigation of exudative macular diseases ${ }^{29}$.

Recently, the OCA method has been combined with an HP-OCT engine ${ }^{30-32}$. This high-penetration optical coherence angiography (HP-OCA) enables vascular imaging of the deep posterior eye.

The purpose of this paper is evaluating the clinical utility of HP-OCA as a non-invasive angiographic modality. We introduce a custom built HP-OCA ${ }^{32}$ to investigate the vascular pathology of exudative macular diseases. A descriptive case series of myopic CNV (mCNV), AMD and PCV are presented. Through detailed discussion of the cases, the clinical utility of HP-OCA is also discussed. 


\section{Subjects and Method}

Eleven eyes of 10 subjects were involved in this study as summaries in Table $1 \mathrm{~s}$. The subjects included 2 eyes of 2 subjects with mCNV, 4 eyes of 4 subjects with AMD, and 5 eyes of 4 subjects with PCV. All subjects were Japanese. The mean age of the patients was $61.3 \pm 14.2$ (mean \pm standard deviation), and the age ranged from 32.8 to 82.3 . Specifically, the mean age of the subjects with each type of disease was 38.8 \pm 8.5 for $\mathrm{mCNV}, 61.9 \pm 5.8$ for AMD and $72.0 \pm 7.5$ for PCV. The mean of the spherical equivalent refractive error of the eyes was $-2.3 \pm 3.8 \mathrm{D}$ ranging from $-11 \mathrm{D}$ to $1.5 \mathrm{D}$. Specifically, the mean refractive error of the eye for each type of disease was $-8.3 \pm 3.9 \mathrm{D}$ for $\mathrm{mCNV}, 0.1 \pm 0.6 \mathrm{D}$ for $\mathrm{AMD}$, and $-1.9 \pm 2.9$

D for PCV.

Table 1. Summary of subjects

\begin{tabular}{|c|c|c|c|c|c|c|}
\hline Patient ID & Eye & Sex & Age & Diopter & Diagnosis & \# of IVR \\
\hline Subject-1 & $\mathrm{R}$ & M & 44.9 & -11.0 & $\mathrm{mCNV}$ & 3 \\
\hline Subject-2 & $\mathrm{L}$ & $\mathrm{M}$ & 32.8 & -5.5 & $\mathrm{mCNV}$ & 0 and 2 \\
\hline Subject-3 & $\mathrm{L}$ & $\mathrm{F}$ & 68.5 & 1.0 & AMD (Classic) & 0 and 3 \\
\hline Subject-4 & $\mathrm{L}$ & $\mathrm{M}$ & 61.5 & 0.0 & AMD (Classic) & 0 and 5 \\
\hline Subject-5 & $\mathrm{L}$ & $\mathrm{M}$ & 63.1 & 0.0 & AMD (Classic) & 0 \\
\hline Subject-6 & $\mathrm{L}$ & $\mathrm{M}$ & 54.4 & -0.5 & AMD (Classic) & 0 and 3 \\
\hline Subject-7 & $\mathrm{L}$ & $\mathrm{M}$ & 82.3 & -0.5 & PCV & 0 \\
\hline \multirow{2}{*}{ Subject- 8} & $\mathrm{R}$ & \multirow{2}{*}{$\mathrm{M}$} & \multirow{2}{*}{71.8} & -5.0 & PCV & 0 \\
\hline & $\mathrm{L}$ & & & -5.0 & PCV & 0 \\
\hline Subject-9 & $\mathrm{R}$ & $\mathrm{M}$ & 68.8 & 1.5 & PCV & 0 \\
\hline Subject-10 & $\mathrm{L}$ & M & 64.9 & -0.5 & PCV & 0 \\
\hline
\end{tabular}

R: right, L: left, M: male, F: female, \# of IVR: number of intravitreal injection of ranibizumab prior to the investigation with HP-OCA. The multiple numbers in the cell of [\# of IVR] represent multiple measurements by HP-OCA after the each number of treatments.

The patients were diagnosed at Ibaraki Medical Center Hospital, Tokyo Medical University. All subjects received a comprehensive ophthalmic examination including color fundus photography, spectral domain OCT with an 830-nm probe (3D OCT-2000, Topcon Corp., Tokyo, Japan), FA, and ICGA. 
Subjects who showed an allergic reaction to fluorescein, one of $\mathrm{mCNV}$ and one of PCV, did not receive FA.

Patients who were diagnosed as exudative macular disease were transferred to an optics laboratory at the University of Tsukuba, where their eyes were scanned with a custom built HP-OCA.

The research protocol adhered to the tenets of the Declaration of Helsinki and was approved by the institutional review board of Tokyo Medical University and the University of Tsukuba. Informed consent was obtained from the subjects after providing an explanation of the nature and possible consequences of the research.

\section{High-penetration Doppler optical coherence angiography}

The HP-OCA device is a prototype built by the Computational Optics Group at the University of Tsukuba. This OCA is based on swept-source OCT technology and offers a measurement speed of 100,000 A-lines/s. With a single scan of the eye, HP-OCA provides both high-penetration OCT and Doppler tomograms. The Doppler tomography signal is obtained by the phase difference between two OCT A-lines, and reflects the Doppler shift of the probe beam. Doppler shift is induced by the flowing red blood cells in the vessels. Based on this mechanism, the Doppler tomography selectively visualizes the ocular vasculature.

This device has two measurement modes for two types of Doppler tomography. The first mode is a bi-directional mode, which calculates the Doppler signal from two successive A-lines. This mode is sensitive to relatively fast flow; in our particular configuration it was from $1.75 \mathrm{~mm} / \mathrm{s}$ to $19.2 \mathrm{~mm} / \mathrm{s}$ (axial velocity) and it provides bi-directional axial flow velocity. A potential drawback of this mode is the relatively high minimum measurable velocity $(1.75 \mathrm{~mm} / \mathrm{s})$. A flow signal slower than this limit is not detectable. In this paper, the bi-directional cross-sectional tomogram, in which the bi-directional Doppler signal overlaid on structural OCT, is denoted as bi-directional Doppler tomogram, while en face projection of the squared bi-directional Doppler tomogram is denoted as bi-directional OCA.

The second mode is the high-sensitive mode, in which the Doppler signal is calculated from two A-lines in two successive B-scans. This mode is sensitive to relatively slow flow, such as $>5.2 \mu \mathrm{m} / \mathrm{s}$ (axial velocity) in our particular configuration. A potential drawback of this mode is relatively low maximum measureable velocity $(60 \mu \mathrm{m} / \mathrm{s})$ for quantitative measurement. A flow signal faster than this limit is wrapped into the measureable Doppler range and appears as a strong but random pattern. Hence this high-sensitive mode is not suitable for qualitative bi-directional measurement, but suitable for high-contrast selective imaging of small vessels. In this mode, the image is displayed in the form of the squared energy of the Doppler signal, and is utilized for small vascular observation with flow-selective contrast. In this paper, a high-sensitive Doppler signal overlaid on structural cross-sectional OCT is denoted as high-sensitive Doppler tomogram, and the en face projection of the high-sensitive Doppler signal is denoted as high-sensitive OCA. 
The details of this HP-OCA are described elsewhere ${ }^{32}$, except for a new signal processing method for eye motion correction. The details of this modification are presented in the supplementary section (S1). It should be noted that the bi-directional mode and high-sensitive mode are denoted as the fast Doppler mode and slow Doppler mode, respectively, in Ref. 32.

The HP-OCA scans the $6 \mathrm{~mm} \times 6 \mathrm{~mm}$ area around the pathologic region with 2048 A-lines (horizontal) $\times 256$ B-scans (vertical) for bi-directional mode and 256 A-lines (horizontal) $\times 2048$ B-scans (vertical) in the high-sensitive mode. Each measurement took 6.6 seconds. It should be noted that a finally obtained en face OCA image of high-sensitive mode is not always perfectly rectangle. This is because of invalid A-lines caused by high-frequency scanning of a scanning mirror in the OCT scanner and its resulting synchronization imperfection between the scanning mirror and data acquisition.

Figure 1 shows typical images of en face OCA (Figure 1e and Figure 1f), OCA tomogram (Figure $1 \mathrm{~g}$ and Figure 1h) and corresponding color fundus (Figure 1a) and ICGA (Figure 1b and Figure 1c) images, which were obtained from a normal macula of a 37-year-old Japanese male with -6.0 D myopia. The en face OCT projection image (Figure 1d) was obtained by the depth average of the logarithmic OCT. Thick choroidal vessels were observed with moderate hyposcattering in the OCT en face projection (Figure 1d); however, the visualization of choroidal vessels were limited compared to the mid-phase of ICGA (Figure 1c). Bi-directional OCA (Figure 1e) and high-sensitive OCA (Figure 1f) showed different vasculatures. The retinal vasculature of the macular region was rarely imaged with bi-directional OCA, while high-sensitive OCA visualized similar retinal vasculature to that of color fundus photograph and the mid-phase of ICGA. For imaging of the choroidal vasculature, the bi-directional OCA showed mainly thick choroidal vasculatures that had fast axial blood flow and appeared in the early-phase of ICGA (Figure 1b), while high-sensitive OCA showed a similar choroidal vasculature to that of the mid-phase of ICGA. In addition, several choroidal vessels that did not appear in the mid-phase ICGA were also visualized in the high-sensitive OCA.

The depth locations of the blood flow are identifiable in Doppler tomogram. In bi-directional Doppler tomogram (Figure 1g), red and blue color represent axial flow directions to the anterior or to the posterior, respectively, and the locations of the flow are indicated with arrows. Figure $1 \mathrm{~h}$ is a vertical high-sensitive Doppler tomogram. The weaving appearance in this Doppler tomogram was caused by imperfect axial motion correction among the horizontal frames. 


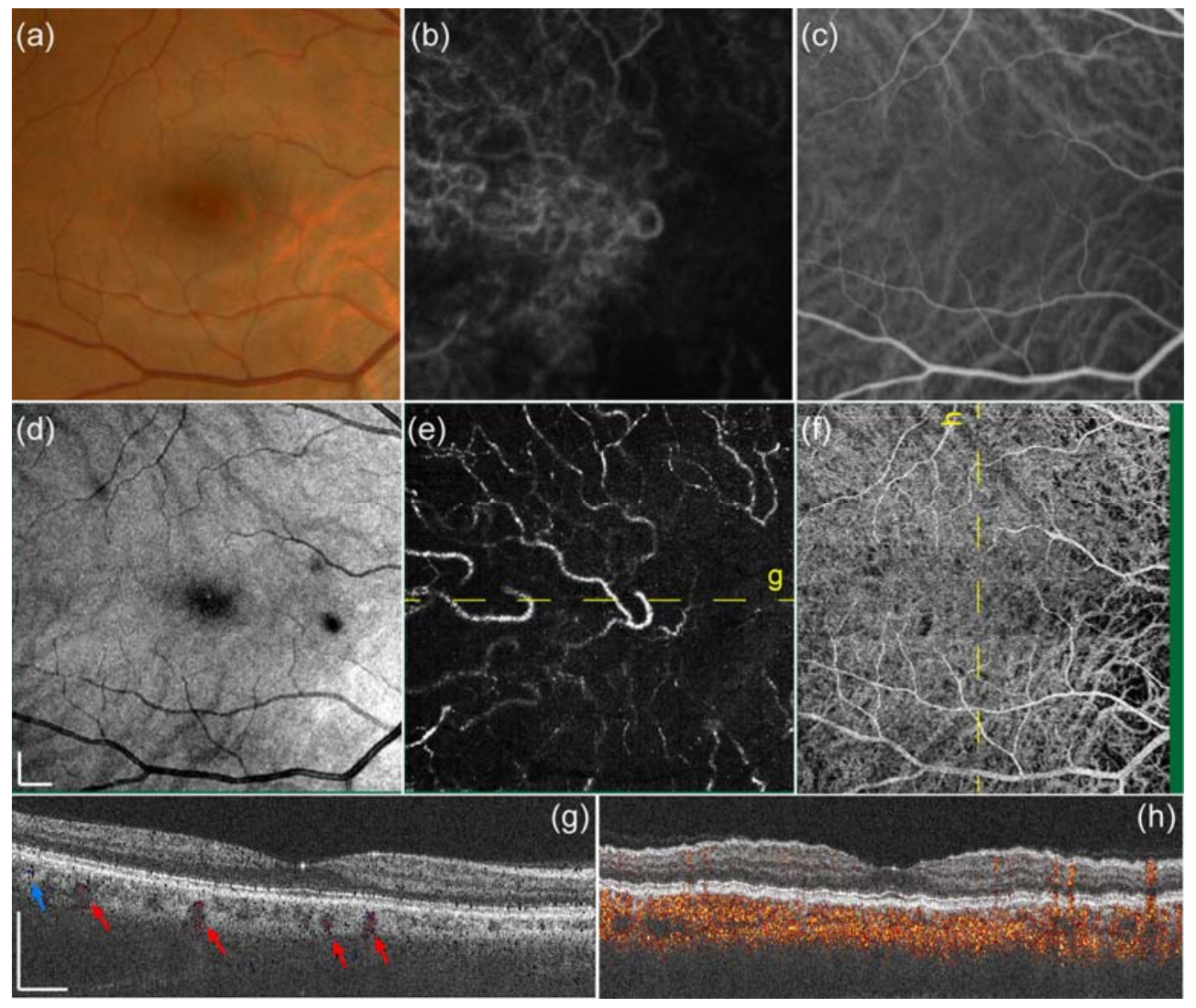

Figure 1. Macular images of a normal eye with $-6.0 \mathrm{D}$ myopia of a 37-year-old Japanese male. (a) is a color fundus photograph, (b) and (c) are early-phase and mid-phase ICGA images, respectively, and (d) is an en face projection of structural OCT. (e) and (f) are bi-directional and high-sensitive OCAs, respectively. (g) and (h) are bi-directional and high-sensitive Doppler tomogram, respectively. Scale bars in (d) and (g) indicate $0.5 \mathrm{~mm}$.

\section{Results}

\section{Case-1: Myopic CNV}

Figure 2 shows a case of mCNV. The subject was a 45 -year-old man who had been received intravitreal ranibizumab injection three times prior to the HP-OCA examination. Figure 2a, Figure $2 b$ and Figure $2 c$ represent the color fundus, FA (late-phase) and ICGA (mid-phase) images, respectively, corresponding to the area of the HP-OCA examination.

RPE elevation was observed in structural OCT (Figure 2f), which was simultaneously obtained with bi-directional tomogram as well as in the high-sensitive Doppler tomogram as indicated by yellow arrows (Figure 2g). In high-sensitive Doppler tomogram, abnormal Doppler signals were observed in the sub-RPE space beneath the elevated RPE and above Bruch's membrane as indicated by a red arrow.

The region of RPE elevation appeared with hyperscattering in en face structural OCT (Figure 2d) and with hyper-Doppler signals in high-sensitive OCA as indicated by arrows (Figure 2e). These signals were 
co-located with the hyperreflective spot in the color fundus (an arrow in Figure 2a) and the hyperfluorescence signal in FA (an arrow in Figure 2b), which is the indicator of inactive CNV. The hyperscattering in the structural OCT projection was surrounded by a hyposcattering rim, as a similar appearance was shown in the color fundus. Similarly, the hyper-Doppler spot in the en face OCA was surrounded by a hypo-Doppler rim, which was similar to a pattern that appeared in the ICGA image (an arrow in Figure 2c). The pattern of CNV appearance in the en face structural OCT and OCA was found in 2 of 2 cases with mCNV, 4 of 4 cases with AMD, and 1 of 4 cases with PCV. It is also noteworthy that high-sensitive OCA showed a high overall similarity to the mid-phase of ICGA.

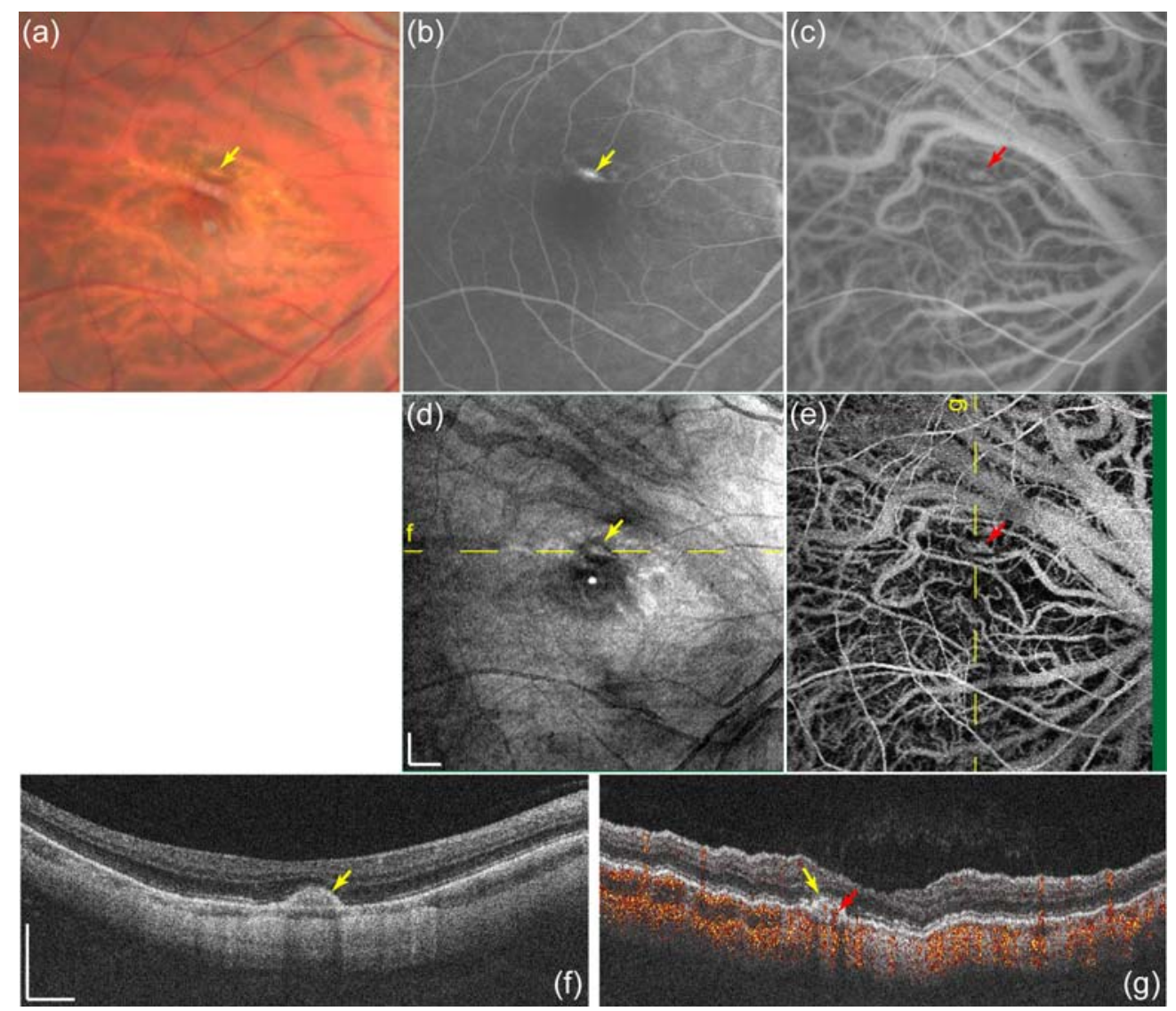

Figure 2. Right macular images of a 45 -year-old man with mCNV, (a) color fundus, (b) FA (late-phase), (c) ICGA (mid-phase), en face projections of (d) structural OCT and (e) high-sensitive OCA, (f) structural OCT taken by a scanning protocol of bi-directional mode and (g) high-sensitive Doppler tomogram. Yellow dashed line in (d) and (e) indicate the location of tomograms of ( $\mathrm{f}$ ) and (g), respectively. Scale bars in (d) and (f) indicate $0.5 \mathrm{~mm}$. A fly-through movie of $(\mathrm{g})$ is available as supplementary of S2. 


\section{Case-2: Myopic CNV with ranibizumab injection}

Figure 3 summarizes another case with mCNV. The subject was a 33-year-old man who was treated with intravitreal ranibizumab injection $(0.05 \mathrm{ml}$ of $10 \mathrm{mg} / \mathrm{ml}$ solution for each injection) two times with a 28-day separation. HP-OCA examination was performed 6 days before the first injection and 59 days after the second injection.

Figure 3a, Figure $3 \mathrm{~b}$ and Figure $3 \mathrm{c}$ are mid-phase ICGA, high-sensitive OCA, and en face structural OCT projection taken before the treatment, respectively. Figure 3d, Figure $3 \mathrm{e}$ and Figure $3 \mathrm{f}$ are corresponding images taken after the injection. The CNV was observed as a hyperfluorescent region surrounded by a dark rim in the ICGA image (red arrow in Figure 3a) before the treatment. This CNV area became smaller after the ranibizumab injection (red arrow in Figure 3d). This CNV appeared as a hyper-Doppler region surrounded by a dark rim in the OCA image (red arrow in Figure 3b), and it also became smaller after the ranibizumab injection (red arrow in Figure 3e). This appearance was well correlated with ICGA. This CNV region appeared as a large hyper-scattering region in en face OCT (a circle in Figure 3c), and this region became smaller after the injection (a circle in Figure 3f).

Figure $3 \mathrm{~g}$ and Figure $3 \mathrm{~h}$ are vertical high-sensitive Doppler tomograhies. These tomograhies were created from the same datasets of Figure $3 \mathrm{~b}$ and Figure 3c, and Figure 3e and Figure 3f, respectively, and the locations of the tomograms are indicated by dashed lines in the en face images. Note that the vertical gap in Figure $3 \mathrm{~g}$ was created by an eye blink. Before the ranibizumab injection, subretinal fluid (blue arrow) and exudates (yellow arrow) were evident in the structural OCT (Figure 3g). The RPE in the exudative region was hardly visible. In addition to structural tomogram, Doppler tomogram provided more detailed insight. Hyper-Doppler signals were observed in the exudates and the choroid beneath the exudates (a red arrow in Figure 3g), which indicated abnormal flow in this region. After ranibizumab injection, as shown in Figure 3h, sub-retinal fluid disappeared and the CNV related elevation became smaller. The RPE became clearly visible with structural OCT. With Doppler tomogram, it was found that the abnormal blood flow disappeared (Figure 3h). 


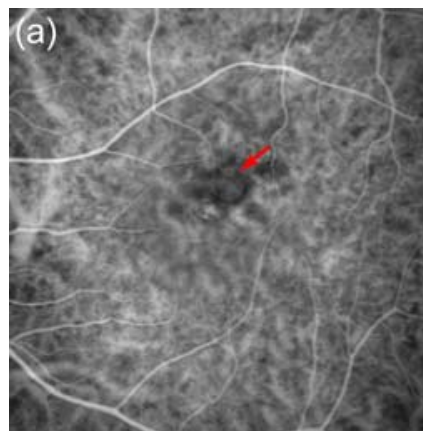

(d)
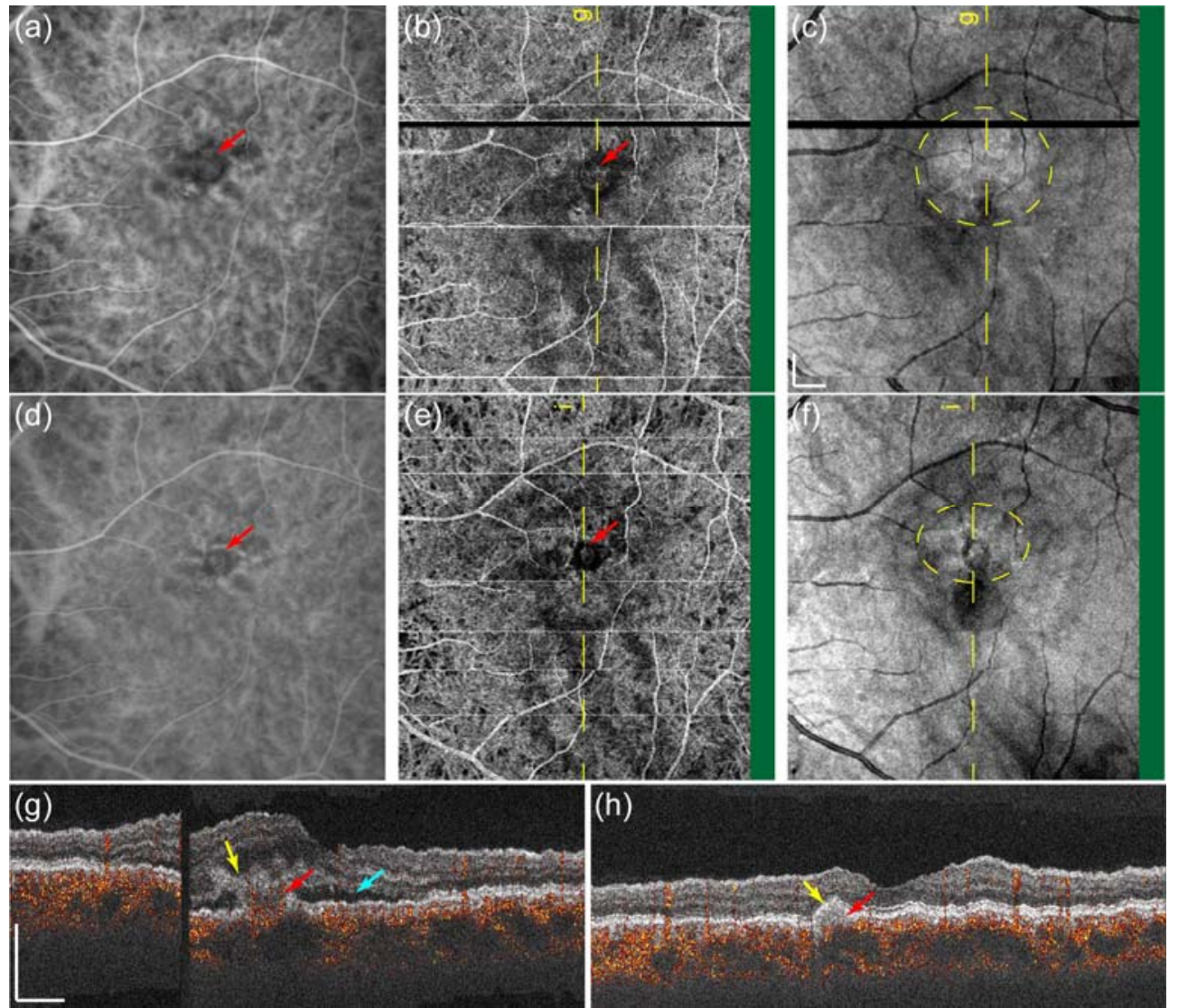

Figure 3. Left macular images of a 33-year-old man with mCNV taken before and after the ranibizumab treatment. (a) and (d) ICGAs (mid-phase), (b) and (e) high-sensitive OCAs, (c) and (f) en face structural OCT projections taken with the high-sensitive scanning mode, and (g) and (i) high-sensitive Doppler tomograms. (a)-(c) and (g) were taken before treatment and (d)-(f) and (i) were taken after treatment. Scale bars in (c) and (g) indicate $0.5 \mathrm{~mm}$. Fly-through movies of (g) and (h) are available as supplementaries of S3 and S4.

\section{Case-3: AMD}

Figure 4 summarizes a case of AMD with classic CNV. The subject was a 62-year-old man. An active $\mathrm{CNV}$ was observed at the fovea by dye leakage in the late-phase of FA (arrow in Figure 4b). The mid-phase of ICGA (Figure 4c) shows CNV that appeared as hyperfluorescence surrounded by a dark rim (arrow). Several hard drusen were observed in the color fundus as white spots (Figure 4a).

OCT en face projection (Figure 4d) shows a similar appearance to the color fundus photograph including hyperscattering spots and choroidal vasculatures. It is noteworthy that the choroidal vasculature around the CNV appeared with hyperscattering signal (red arrow inside a circle), while other choroidal vessels in non-pathologic regions generally appeared with hyposcattering, as indicated by a yellow arrow. This appearance would be caused by the deeper penetration beneath the choroidal vessels around the CNV, as indicated by red arcs in the horizontal structural OCT cross-section taken with the bi-directional 
scanning protocol (Figure 4f).

High-sensitive OCA (Figure 4e) showed almost all of the retinal and choroidal vessels shown in the mid-phase of ICGA ((Figure 4c) even with higher contrast. Remarkably, the detailed abnormal choroidal vasculature was observed at the CNV region, as indicated by a circle in Figure 4e, while it was hardly observed in ICGA.

In high-sensitive Doppler tomogram (Figure 4g), RPE elevation was observed. Remarkably, abnormal Doppler signals were observed beneath the elevated RPE, as indicated by an arrow.

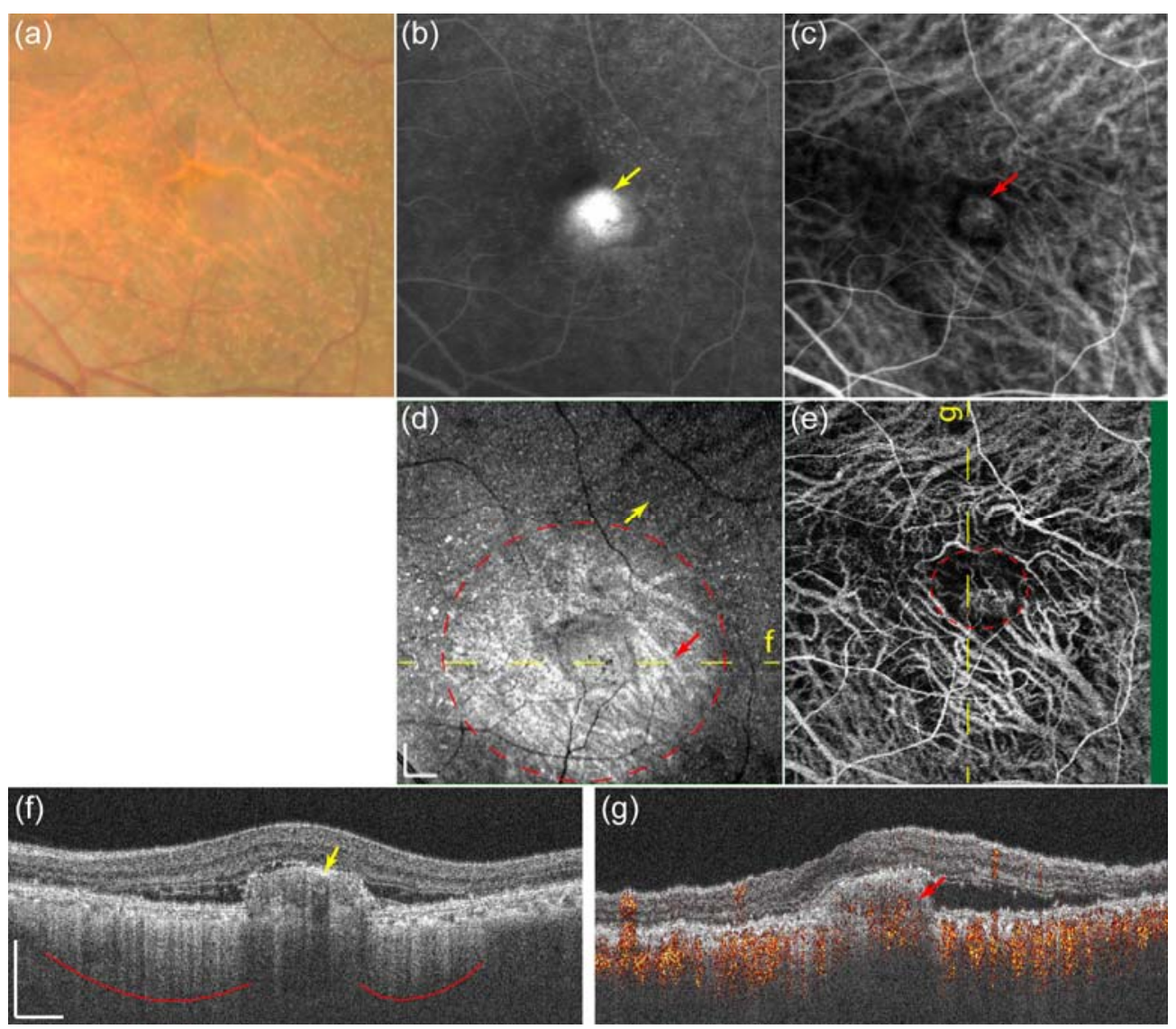

Figure 4. Left macular images of a 62-year-old man with AMD, (a) color fundus, (b) FA (late-phase), (c) ICGA (mid-phase), (d) en face projection of structural OCT, (e) high-sensitive OCA, (f) structural OCT cross-section taken with bi-directional scanning mode, and (g) high-sensitive Doppler tomogram. Yellow dashed lines in (d) and (e) indicate the locations of (f) and (g), respectively. Scale bars in (d) and (f) indicate $0.5 \mathrm{~mm}$. A fly-through movie of $(\mathrm{g})$ is available as supplementary of S5.

\section{Case-4: AMD with ranibizumab injection}

Figure 5 summarizes another case of AMD with classic CNV. The subject was a 69-year-old woman 
treated with intravitreal ranibizumab injection 3 times within time periods of 28 days and 35 days. The first HP-OCA examination was performed 4 days before the first injection and the second examination was done 49 days after the third injection.

Figure 5a-Figure 5d represent late-phase FA, mid-phase ICGA, high-sensitive OCA, and structural OCT projection, respectively, taken before the first injection. Figure 5e-Figure $5 \mathrm{~h}$ are corresponding images taken after the third injection. The active CNV, indicated by a red arrow in the FA image (Figure 5a) became inactive after three injections, as shown in Figure 5e. In the ICGA images, the $\mathrm{CNV}$ appeared as hyperfluorescence surrounded by a dark rim, as indicated by arrows in Figure $5 \mathrm{~b}$ and Figure 5f. The size of the CNV region was reduced by the injections. A similar appearance with ICGA was found in high-sensitive OCA images, as indicated by arrows in Figure $5 \mathrm{c}$ and Figure $5 \mathrm{~g}$, where a hyper-Doppler signal was surrounded by a hypo-Doppler rim. The size of this appearance was also reduced by the ranibizumab injections.

In en face OCT projection (Figure 5d), active CNV appeared with hyperscattering (red arrow). This hyperscattering decreased after the ranibizumab injection (red arrow in Figure 5d). In the post-injection OCT image, some other hyper-scattering spots were observed (yellow arrows in Figure 5h). These hyperscattering spots were well correlated with the hyperfluorescence of FA (arrows in Figure 5e).

A clear difference was observed between the OCT cross-sections taken before and after the injections. Before the treatment (Figure 5i), exudates were observed as indicated by a yellow arrow. In this region, the RPE was not clearly observed. In the corresponding high-sensitive Doppler tomogram, hyper-Doppler signals were observed in the exudates as indicated by a red arrow in Figure 5j. After the injections, exudates disappeared and only a small RPE elevation was observed (yellow arrow in Figure 5k). In high-sensitive Doppler tomogram (Figure 51), nearly no abnormal Doppler signal was observed (arrow). 


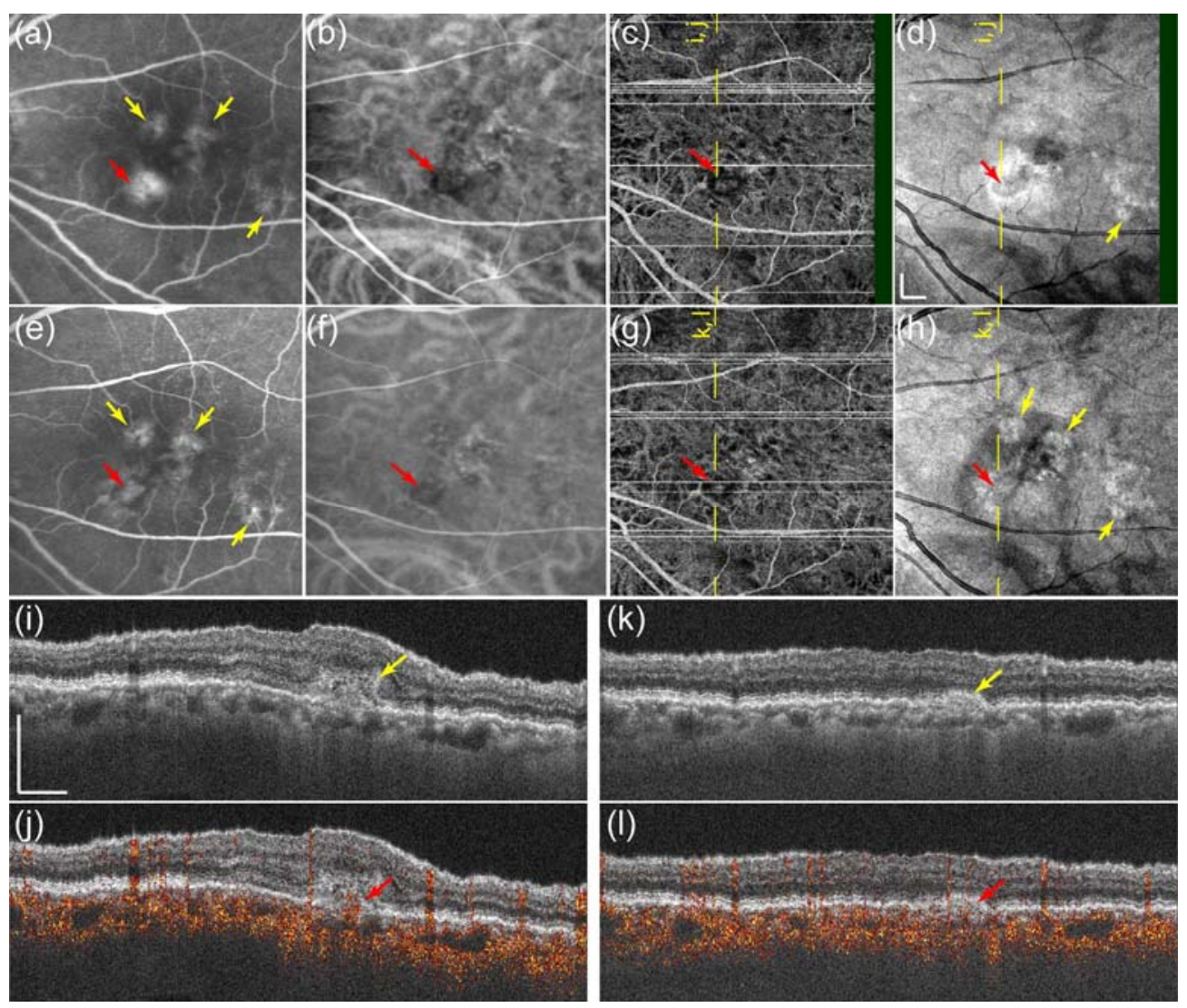

Figure 5. Left macular images of a 69-year-old woman with AMD taken before and after the three times of intravitreal ranibizumab injection. (a) FA (late-phase), (b) ICGA (mid-phase), (c) high-sensitive OCA, and (d) en face structural OCT projection images taken using the high-sensitive scanning mode before the first ranibizumab injection. (e)-(h) are corresponding images taken after the third ranibizumab injection. (i) and (j) are structural OCT and high-sensitive Doppler tomogram taken before the first injection. The locations of these tomograms are indicated by yellow lines in (c) and (d). (k) and (l) are those taken after the third injection. The yellow lines in $(\mathrm{g})$ and $(\mathrm{h})$ indicate the location of these tomograms. Scale bars in (d) and (i) indicate $0.5 \mathrm{~mm}$. Fly-through movies of (j) and (1) are available as supplementaries of S6 and S7.

\section{Case-5: PCV}

Figure 6 summarizes a case of PCV that appeared with a large abnormal vascular network. The subject was a 65 -year-old man and his left eye was examined. The eye was not treated at the time of the examination. Widely spread exudates were observed with white color in the fundus photograph (Figure 6a). The hyperfluorescent region in the late-phase FA (Figure 6g) indicated a large pigment epithelial detachment (PED). Abnormal vasculature and polypoids were observed in the mid-phase of ICGA (Figure 6c). In en face OCT projection (Figure 6d), a strong hyperscattering (left circle) and a moderate 
hyper-scattering (right circle) were observed. This corresponded to the hyperfluorescence regions in FA (Figure 6g).

In bidirectional Doppler tomogram (Figure 6h), clear Doppler signals were observed beneath the moderate PED (red arrow). Figure 6e shows the bi-directional OCA, where the red arrow indicates the Doppler signal in Figure 6h. When comparing this OCA and early phase ICGA (Figure 6b), it is evident that this Doppler signal corresponds to a feeder vessel.

In high-sensitive Doppler tomogram (Figure 6i), many abnormal Doppler signals were observed beneath the PED as indicated by the red arrows. Remarkably, some Doppler signals, such as that indicated by the leftmost arrow, represented abnormal vessels that penetrated Bruch's membrane into the sub-RPE space. The high-sensitive OCA (Figure 6f) shows a highly correlated pattern with the mid-phase of ICGA (Figure 6c). 


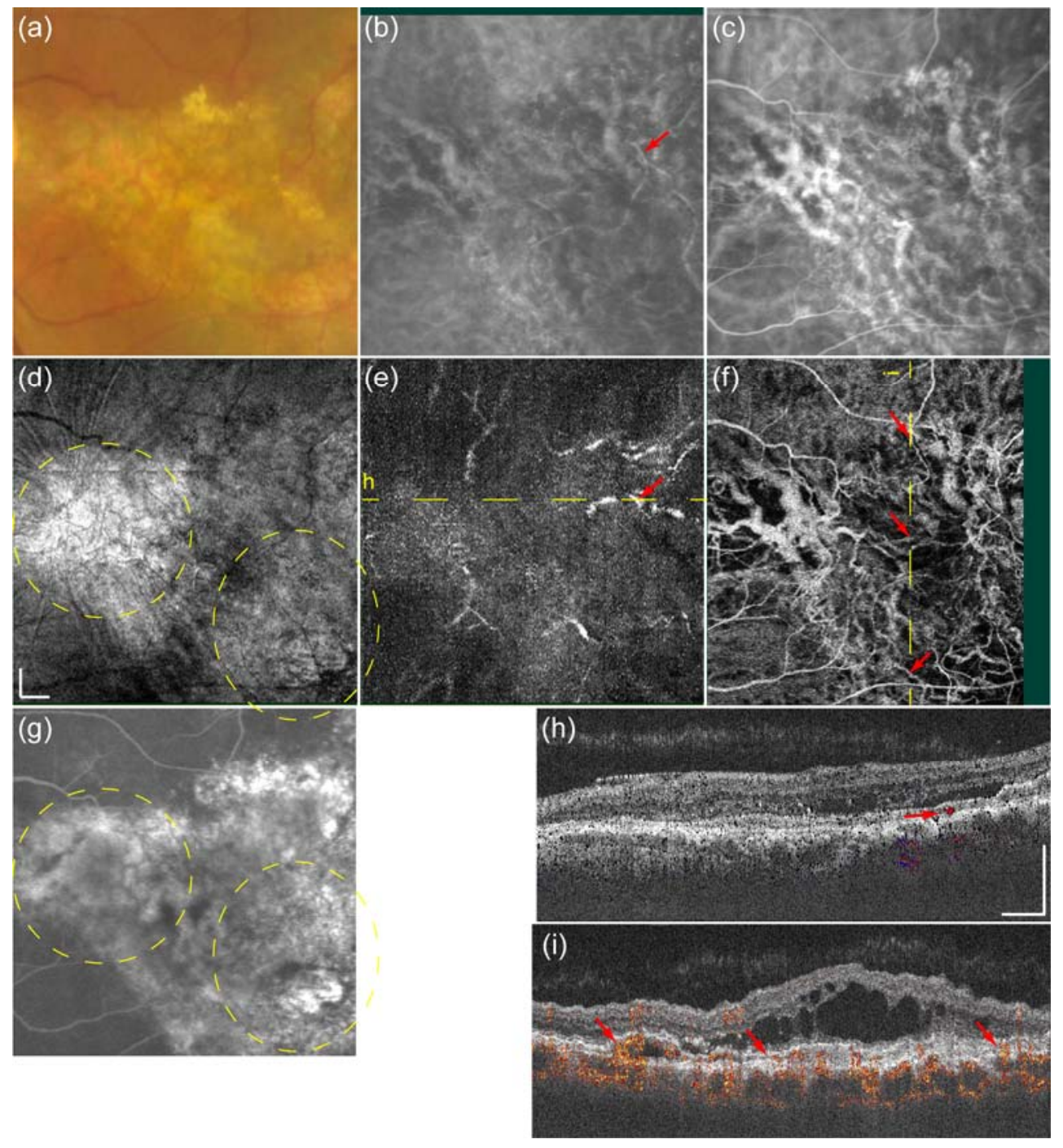

Figure 6. Left macular images of a 65-year-old man with PCV with a large area of polypoid, (a) color fundus photograph, (b) early-phase of ICGA, (c) mid-phase of ICGA, (d) en face projections of structural OCT, (e) bi-directional OCA, (f) high-sensitive OCA, (g) late-phase of FA, (h) bi-directional Doppler tomogram, and (i) high-sensitive Doppler tomogram. Yellow dashed lines in (e) and (f) indicate the location of (h) and (i), respectively. Scale bars in (d) and (h) indicate $0.5 \mathrm{~mm}$. Fly-through movies of (h) and (i) are available as supplementaries of S8 and S9.

\section{Case-6: PCV}

Figure 7 summarizes another case of PCV. The subject was a 69-year-old man. The early-phase of ICGA (Figure 7a) shows a clear branching vascular network (BVN) at the PED area. The late-phase of ICGA (Figure 7d) shows terminal aneurismal dilatation of the BVN. High-sensitive OCA (Figure 7b) shows a highly correlated vascular pattern including the BVN with the early-phase of ICGA. High-sensitive 
Doppler tomogram (Figure 7g) presents several Doppler signals beneath the PED (arrows). The location of this Doppler tomogram is indicated on ICGAs (Figure 7a and Figure 7d) and en face OCA (Figure 7b) with yellow lines. This indicates that the Doppler signals beneath the PED in Figure $7 \mathrm{~g}$ are associated with the BVN.

A volume rendering of the high-sensitive OCA provides more intuitive understanding, as shown in Figure 7e, where the Doppler signal and the structural OCT signal are respectively displayed in orange and green colors. In this image, the comprehensive three-dimensional structure of the BVN is clearly visualized. In addition, a blood vessel connecting the BVN to the choroid is clearly observed as indicated with an arrow.

An active $\mathrm{CNV}$ is observed in FA, as indicated by a circle in Figure 7c. A similar pattern is observed in high-sensitive OCA (Figure 7b) and OCT en face projection (Figure 7f) with hyper-Doppler and hyperscattering, respectively. Corresponding Doppler signals are observed in the choroid under the PED (arrow in Figure 7h).

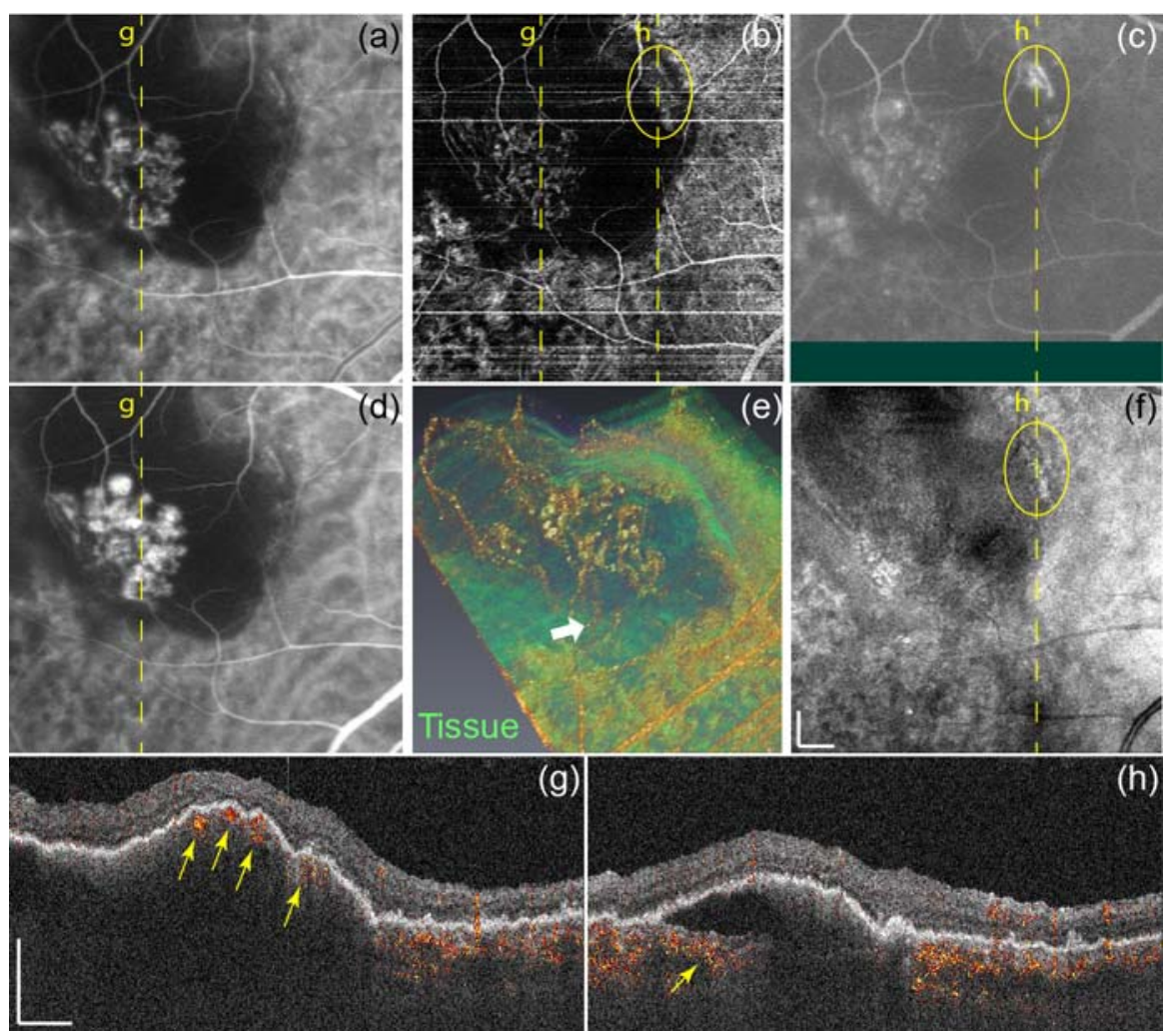

Figure 7. Right macular images of a 69-year-old man with PCV with a clear BVN. (a) early-phase and (d) late-phase ICGA, (b) high-sensitive OCA, (e) volume rendering of high-sensitive three-dimensional Doppler tomogram, (c) late-phase FA, (f) en face projection of structural OCT. (g) and (h) are high-sensitive Doppler tomograms. The location of $(\mathrm{g})$ is indicated by dashed lines in (a), (b), and (d), while the location of (h) is indicated by yellow lines in (b), (c), and (f). Scale bars in (f) and (g) indicate $0.5 \mathrm{~mm}$. A 
volume rendering movie of (e) and a combined fly-through movie of (g) and (h) are available as supplementaries of S10 and S11.

\section{Visibility of Doppler signals at abnormal choroidal vessels}

Two graders (YJH and MM) graded the visibility of abnormal Doppler signals. A fly-through sequence of structural OCT, Doppler tomogram, and the Doppler tomogram overlaid on the structural OCT were presented to the graders. In this grading, the eyes with Doppler signals at abnormal regions found in structural OCT were labeled as positive and the other eyes were labeled as negative. This grading was independently done for the following three regions; (1) an abnormal region anterior to the RPE, (2) a region between the RPE and Bruch's membrane, and (3) within Bruch's membrane, i.e., Doppler signal penetrated through Bruch's membrane.

Table 2 summarizes the occurrence of Doppler signals at abnormal choroidal vessels graded by two graders and their agreements. In this table, the three regions described above were denoted as "above RPE," "RPE-Bruch," and "in Bruch." The three numbers in each cell represent, from the left, the number of positive eyes found by grader $\mathrm{YJH}$, that found by grader $\mathrm{MM}$, and the number of eyes in which the grading of the two graders are agreed.

With bi-directional Doppler tomogram, abnormal flows were observed in PCV eyes with $80 \%$ by grader YJH and 60\% by MM. Conversely, no abnormal flow was observed in mCNV and AMD cases. High-sensitive OCA showed a high occurrence of abnormal Doppler signals within the untreated eyes with both graders. The grading of the two graders were perfectly agreed except the following three cells; the bi-directional Doppler of untreated PCV at the "RPE-Bruch" and "in Bruch" region, high-sensitive Doppler of untreated PCV at the "In Bruch" region, and high-sensitive Doppler of mCNV at the "RPE-Bruch" region. And hence, the overall agreement of all cells in

Table 2 was $89 \%(82 / 90)$.

Table 2. Visibility of Doppler signal at abnormal choroidal vessels graded by two graders and their agreement

\begin{tabular}{|c|c|c|c|c|c|c|}
\hline \multirow{2}{*}{$\begin{array}{c}\text { Doppler } \\
\text { mode }\end{array}$} & \multirow{2}{*}{ Region } & $\begin{array}{c}|c| \\
\mathrm{mCNV} \\
(\mathrm{N}=1)\end{array}$ & $\begin{array}{c}\text { AMD } \\
(\mathrm{N}=4)\end{array}$ & $\begin{array}{c}\mathrm{PCV} \\
(\mathrm{N}=5)\end{array}$ & $\begin{array}{c}\mathrm{mCNV} \\
(\mathrm{N}=2)\end{array}$ & $\begin{array}{c}\text { AMD } \\
(\mathrm{N}=3)\end{array}$ \\
\hline \multirow{2}{*}{$\begin{array}{c}\text { Bi- } \\
\text { directional } \\
\text { mode }\end{array}$} & Above RPE & $0,0,1$ & $0,0,4$ & $0,0,5$ & $0,0,2$ & $0,0,3$ \\
\cline { 2 - 7 } & RPE-Bruch & $0,0,1$ & $0,0,4$ & $4,3,4$ & $0,0,2$ & $0,0,3$ \\
\cline { 2 - 7 } & In Bruch & $0,0,1$ & $0,0,4$ & $2,1,2$ & $0,0,2$ & $0,0,3$ \\
\hline High- & Above RPE & $1,1,1$ & $3,3,4$ & $0,0,5$ & $0,0,2$ & $0,0,3$ \\
\hline
\end{tabular}




\begin{tabular}{|c|c|c|c|c|c|c|}
\hline \multirow{2}{*}{$\begin{array}{c}\text { sensitive } \\
\text { mode }\end{array}$} & RPE-Bruch & $0,0,1$ & $1,1,4$ & $5,5,5$ & $1,2,1$ & $1,2,3$ \\
\cline { 2 - 7 } & In Bruch & $1,1,1$ & $4,4,4$ & $4,5,4$ & $1,1,2$ & $1,2,3$ \\
\hline
\end{tabular}

A summary occurrence of Doppler signals in bi-directional and high-sensitive Doppler tomograms. The values in each cell, from the left, represent the number of eyes in which the Doppler signal was found by grader $\mathrm{YJH}$, that found by MM, and agreement between two graders. $N$ is the number of eyes. Three regions are defined as: (1) an abnormal region anterior to the RPE (above the RPE), (2) a region between the RPE and Bruch's membrane (RPE-Bruch), and (3) within Bruch's membrane (in Bruch), in which case a Doppler signal penetrated through Bruch's membrane

\section{Discussion}

In the en face OCT projections of 1 eye with AMD (Figure 4d) and 2 eyes of a single case of PCV, the choroidal vessels appeared with hyperscattering at the pathologic regions, while it appeared with hyposcattering in non-pathologic regions. It is also known that, the choroidal vessels appeared with hyposcattering in the en face OCT projection of normal eyes. In these pathologic regions, abnormal hyperpenetration to the deep choroid and sclera was observed as exemplified in Figure 4f. This hyperscattering could be explained by abnormality in the choroidal or RPE tissue, such as reduction of melanin content, abnormal thinning of the choroid or both. The hyposcattering appearance of choroidal vessels can be utilized as an indicator of the choroidal abnormality.

Since the bidirectional mode is only sensitive to fast blood flow, it is selectively sensitive to the arteries among the choroidal vessels. This property of the bidirectional mode provides a similarity between bidirectional OCA and the early-phase (arterial phase) of ICGA as exemplified in Figure $1 \mathrm{~b}$ and Figure 1e. For ICGA imaging of the PCV, feeder vessels were imaged at the early phase. It would be expected that the feeder vessels can be visualized by bidirectional OCA. In the current study, bidirectional OCA visualized choroidal vessels beneath or close to the PED in 3 of 5 PCV eyes to both graders. These choroidal vessels were correlated with the feeder vessels that appeared in early-phase ICGA. This indicates the utility of bidirectional OCA for noninvasive imaging of feeder vessels of PCV.

The high-sensitive OCA of PCV cases visualized the three-dimensional (3-D) structure of the BVN and polypoids. It is known that a cluster of abnormal vessels are co-localized in some region beneath the PED and a feeder vessel connects the abnormal vessel cluster and a choroidal vessel ${ }^{7}$.

The abnormal vessel cluster of PCV has been investigated by structural OCT with a $830-\mathrm{nm}$ probe beam $^{7}$, structural high-penetration OCT with a $1-\mu \mathrm{m}$ probe beam ${ }^{13}$, and Doppler OCT with a $840-\mathrm{nm}$ probe beam ${ }^{29}$. However, none of them has been used to visualize the comprehensive 3-D structure of PCV including the vessels cluster and the feeder vessel. As exemplified by Figure 7d, high-sensitive OCA has enabled comprehensive visualization of the 3-D structure of the PCV including the vessel cluster and the feeder vessel. This comprehensive investigative ability of high-sensitive OCA can provide a detailed 
understanding of the pathology.

The appearance of a high-sensitive Doppler en face projection image is generally well correlated with mid-phase ICGA, as exemplified in Figure 1c and 1f, Figure 2c and 2e, Figure 3a and 3b, Figure 3d and e, Figure $4 \mathrm{c}$ and $4 \mathrm{e}$, Figure $5 \mathrm{~b}$ and $5 \mathrm{c}$, Figure $5 \mathrm{f}$ and $5 \mathrm{~g}$, and Figure $6 \mathrm{c}$ and $6 \mathrm{f}$. The CNV appearance with high-sensitive OCA was also similar to that of ICGA. Furthermore, in some cases, such as shown in Figure 4, Figure 6, and Figure 7, high-sensitive OCA provided higher contrast than ICGA.

In the current study, FA images showed leakages in some untreated cases including 1 eye with mCNV, 4 eyes with AMD and 1 eye with PCV. The number of leakages was 6 in total. In all leakage spots, a similar pattern was observed in en face high-sensitive OCA as exemplified in Figure 2b and 2e, Figure $4 \mathrm{~b}$ and $4 \mathrm{e}$, Figure $5 \mathrm{a}$ and $5 \mathrm{c}$, and Figure $7 \mathrm{c}$ and $7 \mathrm{~b}$. The leakage in FA is an indicator of abnormal penetration of a choroidal vessel into the retina, while the Doppler OCT, and hence OCA, is sensitive to localized motion and selectively contrasts the structure of the blood vessels. Although, the leakage is not directly detectable by OCA, its related structural abnormality can be detected. And hence, high-sensitive OCA has indirectly indicated the region of the leakage. Because of the difference in the contrast mechanism, OCA cannot be the full alternative for FA. However, the high correlation between OCA and FA and non-invasive nature of OCA would make OCA a comparable utility to FA.

While FA and ICGA use fluorescent dye as a vessel contrast agent, HP-OCA uses blood flow as a contrast source. The different vessel contrast mechanism between conventional angiography and HP-OCA would result in different characteristics. FA and ICGA have advantages of time elapse imaging after dye injection and investigation of tissue damage through dye leakage. Conversely, OCA has the two major advantages of 3-D investigation and non-invasiveness. The 3-D visualization of the ocular vasculature would provide more detailed insight of the pathology. The non-invasiveness enables safe and repetitive application of OCA to the patient. This repetitive examination is particularly useful for the frequent monitoring of the outcome of treatments. This could provide insights in natural history and response to the treatment of retinal illness. In addition, OCA can be also utilized for mass-screening not only for the specific clinical diagnosis and the time-sequence monitoring.

Since HP-OCA provides vast amount of information including a structural OCT volume and two types of Doppler OCT volumes, a conventional static-page-oriented patient report is not always convenient for clinicians. Alternatively, a sophisticated data browser is necessary. Figure 8 shows a screen shot of our custom made HP-OCA browser. It consists of an en face projection image of structural OCT and en face Doppler OCA in its left column, while cross-sectional images of structural OCT, Doppler tomogram, and the Doppler tomogram overlaid on the structural OCT are displayed at the right column. An operator is allowed to quickly find the point of pathology in the en face images. By pointing the position of pathology in the en face images with a line cursor, corresponding cross-sections are displayed in the right column. Hence the operator can utilize both the structural OCT projection and en face OCA to quickly find the pathologic region. This simultaneous usage of the structural projection and en face OCA 
reduces the risk of overlooking of pathologies in comparison to a conventional OCT. The operator is also allowed to markup one of the cross-sectional images. As marking up an image, the same mark simultaneously appears at the same location in the other cross-sectional images. This function enables quick co-registration of structural and Doppler findings. By using a well-designed data browser, such as this HP-OCA browser, the operator can quickly and effectively review the huge amount of information provided by HP-OCA.

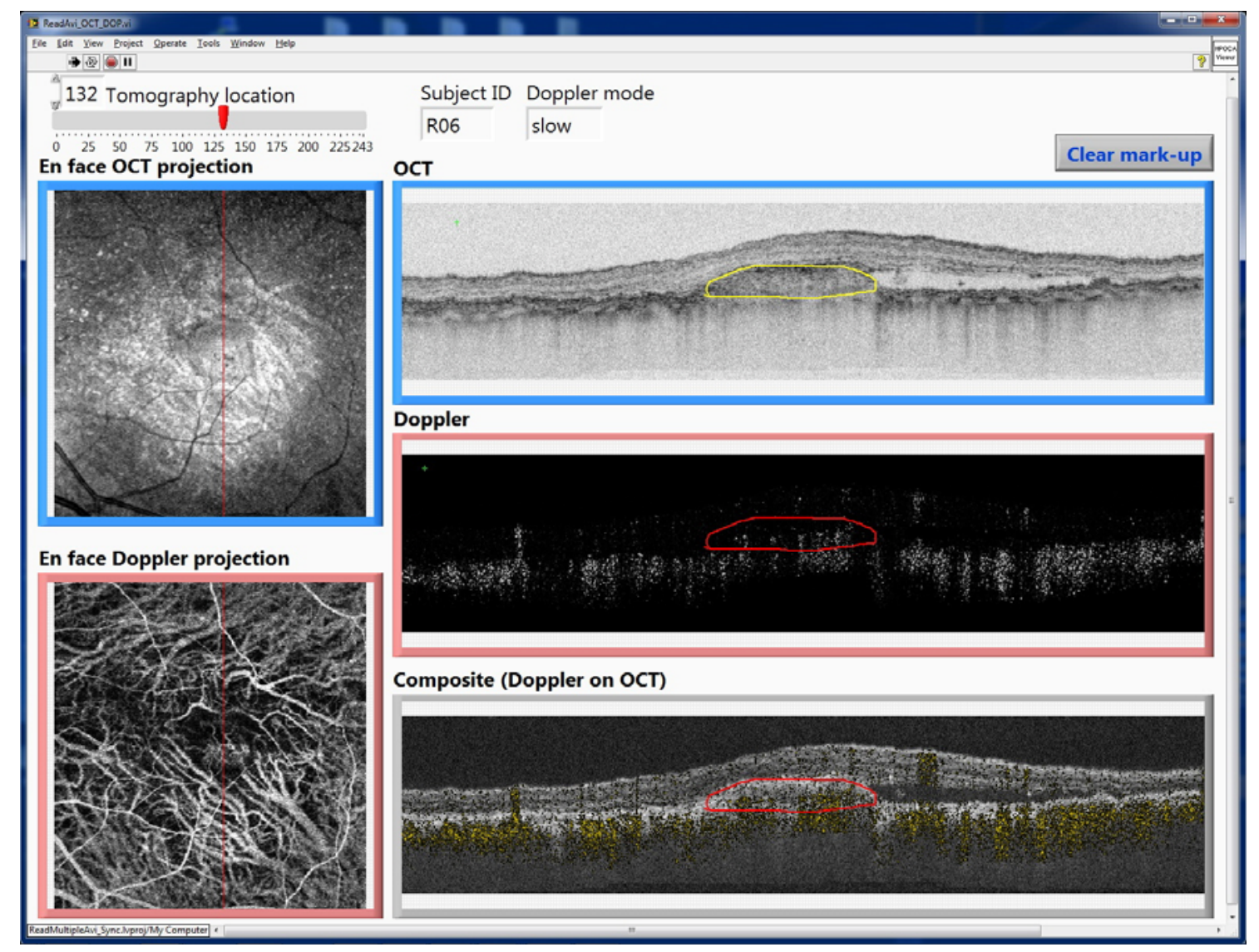

Figure 8. A screen shot of a custom-made HP-OCA data browser. The en face projection of structural OCT and en face Doppler OCA are displayed at the left column and the cross-sectional structural OCT, Doppler tomogram, and the Doppler tomogram overlaid on the structural OCT are displayed in the right column. As pointing the position of interest in the en face images by a red line cursor, the corresponding cross-sectional images are simultaneously displayed in the right column. The operator can mark up one of the cross-sections, and the same mark simultaneously appears at the same positions of the other cross-sections.

In this paper, we demonstrated vasculature imaging of exudative macular diseases by 
HP-OCA. HP-OCA provided two measurement modes; i.e. bidirectional OCA and high-sensitive OCA. With bi-directional OCA, feeder vessels of the PCV were successfully visualized. Conversely, the high-sensitive OCA provided similar angiograms to the mid-phase of ICGA but with higher contrast. This new modality can be partially utilized as an alternative to FA and ICGA. In addition, the non-invasive and 3-D imaging ability of OCA enables wider applications than FA and ICGA.

\section{Acknowledgements}

This study was supported by the Japan Science and Technology Agency through a contract from the Development of Systems and Technology for Advanced Measurement and Analysis, and also supported in part by the Japan Society for the Promotion of Science (JSPS) KAKENHI Grant Number 11J01600, 24592682. Young-Joo Hong is supported by the Research Fellowships of the JSPS for Young Scientists.

\section{References}

1. Yannuzzi LA. The retinal atlas. Philadelphia: Saunders/Elsevier; 2010.

2. Ikuno Y, Tano Y. Retinal and Choroidal Biometry in Highly Myopic Eyes with Spectral-Domain Optical Coherence Tomography. Invest. Ophthalmol. Vis. Sci. 2009;50(8):3876-3880.

3. Ikuno Y, Jo Y, Hamasaki T, Tano Y. Ocular Risk Factors for Choroidal Neovascularization in Pathologic Myopia. Invest. Ophthalmol. Vis. Sci. 2010;51(7):3721-3725.

4. Bressler NM, Bressler SB, Fine SL. Age-related macular degeneration. Surv. Ophthalmol. 1988;32(6):375- 413 .

5. AREDS. Risk factors associated with age-related macular degeneration: A case-control study in the age-related eye disease study: age-related eye disease study report number 3. Ophthalmology. 2000;107(12):2224- 2232.

6. Yannuzzi LA, Wong DWK, Sforzolini BS, et al. Polypoidal Choroidal Vasculopathy and Neovascularized Age-related Macular Degeneration. Arch. Ophthalmol. 1999;117(11):1503-1510.

7. Tsujikawa A, Sasahara M, Otani A, et al. Pigment epithelial detachment in polypoidal choroidal vasculopathy. Am. J. Ophthalmol. 2007;143(1):102-111.

8. Yannuzzi LA, Rohrer KT, Tindel LJ, Sobel R, Costanza M. Fluorescein angiography complication survey. Ophthalmology. 1986;93:611-617.

9. Hope-Ross M, Yannuzzi LA, Gragoudas ES, et al. Adverse reactions due to indocyanine green. Ophthalmology. 1994;101:529-533. 
10. Huang D, Swanson E, Lin C, et al. Optical coherence tomography. Science. 1991;254(5035):1178-1181.

11. Unterhuber A, Považay B, Hermann B, Sattmann H, Chavez-Pirson A, Drexler W. In vivo retinal optical coherence tomography at $1040 \mathrm{~nm}$ - enhanced penetration into the choroid. Opt. Express. 2005;13(9):3252-3258.

12. De Bruin DM, Burnes DL, Loewenstein J, et al. In Vivo Three-Dimensional Imaging of Neovascular Age-Related Macular Degeneration Using Optical Frequency Domain Imaging at $1050 \mathrm{~nm}$. Invest. Ophthalmol. Vis. Sci. 2008;49(10):4545-4552.

13. Yasuno Y, Miura M, Kawana K, et al. Visualization of Sub-retinal Pigment Epithelium Morphologies of Exudative Macular Diseases by High-Penetration Optical Coherence Tomography. Invest. Ophthalmol. Vis. Sci. 2009;50(1):405-413.

14. Wang XJ, Milner TE, Nelson JS. Characterization of fluid flow velocity by optical Doppler tomography. Opt. Lett. 1995;20(11):1337-1339.

15. Chen Z, Milner TE, Srinivas S, et al. Noninvasive imaging of in vivo blood flow velocity using optical Doppler tomography. Opt. Lett. 1997;22(14):1119-1121.

16. Zhao Y, Chen Z, Saxer C, Xiang S, De Boer JF, Nelson JS. Phase-resolved optical coherence tomography and optical Doppler tomography for imaging blood flow in human skin with fast scanning speed and high velocity sensitivity. Opt. Lett. 2000;25(2):114-116.

17. Zhao Y, Chen Z, Saxer C, et al. Doppler standard deviation imaging for clinical monitoring of in vivo human skin blood flow. Opt. Lett. 2000;25(18):1358-1360.

18. Leitgeb RA, Schmetterer L, Drexler W, Fercher AF, Zawadzki RJ, Bajraszewski T. Real-time assessment of retinal blood flow with ultrafast acquisition by color Doppler Fourier domain optical coherence tomography. Opt. Express. 2003;11:3116-3121.

19. White B, Pierce M, Nassif N, et al. In vivo dynamic human retinal blood flow imaging using ultra-high-speed spectral domain optical coherence tomography. Opt. Express. 2003;11(25):3490-3497.

20. Leitgeb RA, Schmetterer L, Hitzenberger CK, et al. Real-time measurement of in vitro flow by Fourier-domain color Doppler optical coherence tomography. Opt. Lett. 2004;29(2):171-173.

21. Wehbe H, Ruggeri M, Jiao S, Gregori G, Puliafito CA, Zhao W. Automatic retinal blood flow calculation using spectral domain optical coherence tomography. Opt. Express. 2007;15(23):15193. 
22. Makita S, Fabritius T, Yasuno Y. Quantitative retinal-blood flow measurement with three-dimensional vessel geometry determination using ultrahigh-resolution Doppler optical coherence angiography. Opt. Lett. 2008;33(8):836-838.

23. Wang Y, Bower BA, Izatt JA, Tan O, Huang D. Retinal blood flow measurement by circumpapillary Fourier domain Doppler optical coherence tomography. J. Biomed. Opt. 2008;13(6):064003.

24. Szkulmowska A, Szkulmowski M, Szlag D, Kowalczyk A, Wojtkowski M. Three-dimensional quantitative imaging of retinal and choroidal blood flow velocity using joint Spectral and Time domain Optical Coherence Tomography. Opt. Express. 2009;17(13):10584-10598.

25. Makita S, Hong Y, Yamanari M, Yatagai T, Yasuno Y. Optical coherence angiography. Opt. Express. 2006;14(17):7821-7840.

26. An L, Wang RK. In vivo volumetric imaging of vascular perfusion within human retina and choroids with optical micro-angiography. Opt. Express. 2008;16(15):11438.

27. Kim DY, Fingler J, Werner JS, Schwartz DM, Fraser SE, Zawadzki RJ. In vivo volumetric imaging of human retinal circulation with phase-variance optical coherence tomography. Biomed. Opt. Express. 2011;2(6):1504-1513.

28. Makita S, Jaillon F, Yamanari M, Miura M, Yasuno Y. Comprehensive in vivo micro-vascular imaging of the human eye by dual-beam-scan Doppler optical coherence angiography. Opt. Express. 2011;19(2):1271-1283.

29. Miura M, Makita S, Iwasaki T, Yasuno Y. Three-Dimensional Visualization of Ocular Vascular Pathology by Optical Coherence Angiography In Vivo. Inv. Ophthalmol. Vis. Sci. 2011;52(5):2689 -2695.

30. Baumann B, Potsaid B, Kraus MF, et al. Total retinal blood flow measurement with ultrahigh speed swept source/Fourier domain OCT. Biomed. Opt. Express. 2011;2(6):1539-1552.

31. Braaf B, Vermeer KA, Sicam VADP, Van Zeeburg E, Van Meurs JC, De Boer JF. Phase-stabilized optical frequency domain imaging at $1-\mu \mathrm{m}$ for the measurement of blood flow in the human choroid. Opt. 2011;19(21):20886-20903.

32. Hong Y-J, Makita S, Jaillon F, et al. High-penetration swept source Doppler optical coherence angiography by fully numerical phase stabilization. Opt. Express. 2012;20(3):2740-2760.

33. Kasai C, Namekawa K, Koyano A, Omoto R. Real-Time Two-Dimensional Blood Flow Imaging Using an Autocorrelation Technique. Sonics and Ultrasonics, IEEE Transactions on. 1985;32(3):458 - 464. 
34. Yang VXD, Gordon ML, Mok A, et al. Improved phase-resolved optical Doppler tomography using the Kasai velocity estimator and histogram segmentation. Opt. Commun. 2002;208(4-6):209-214.

\section{Supplementary material}

\section{Modified Phase-II numerical phase stabilization}

In this study, we employed a custom-made HP-OCA system. Most of the details have been described in Ref. 32, except for an updated algorithm for Doppler tomography reconstruction. HP-OCA relies on software based phase stabilization algorithms, called phase-1 and phase- 2 phase stabilizations. The phase-1 stabilization cancels the phase error of OCT that occurred because of system instability. The phase-2 stabilization is for fine cancelation of system-induced phase error and also for cancelation of the offset Doppler shift that occurred with bulk motion of the eye. The HP-OCA system employed in this study uses an updated version of the phase-2 stabilization algorithm.

The details of the original version of the phase- 2 stabilization algorithm is described in Section 2.3 of Ref. 32. The core process of this algorithm is iterative weighted regression of the measured depth-resolved Doppler shift signal. In the original algorithm, the weight was updated according to the following equations for each iteration (Eqs. (11) and (12) of Ref. 34).

$$
\begin{gathered}
W_{m}(\zeta)=\left\{\begin{array}{ccc}
\sqrt{I(\zeta)} & : & I(\zeta)>\varepsilon^{2} \\
0 & : & \text { otherwise }
\end{array} \text { for } m=0\right. \\
W_{m}(\zeta)=\left\{\begin{array}{ccc}
0 & :\left|\Delta \varphi(\zeta)-\left(a_{m-1} \zeta+b_{m-1}\right)\right|>\pi /(2 m) & \text { otherwise } \\
W_{m-1}(\zeta) & : & \text { for } m \geq 1
\end{array}\right.
\end{gathered}
$$

where $m=0,1, \cdots, M$ is the index of iteration with $M$ of the maximum iteration index, $W_{m}(\zeta)$ is fitting weight of $m$-th iteration with $\zeta$ as the Fourier pair of wavenumber. $I(\zeta)$ is signal intensity of OCT, $\varepsilon^{2}$ is the noise level of the OCT. $\Delta \varphi(\zeta)$ is the measured Doppler phase signal corrected by phase-1 stabilization algorithm and $a_{m-1} \zeta+b_{m-1}$ is a linear fitting of the phase at $(m-1)$-th iteration.

In the updated algorithm, the Eq. (2) was revised to be

$$
W_{m}(\zeta)=\left\{\begin{array}{ccc}
0 & : & \left|\Delta \varphi(\zeta)-\left(a_{m-1} \zeta+b_{m-1}\right)\right| \geq T(m ; p, M) \\
W_{0}(\zeta) & : & \text { otherwise }
\end{array}\right.
$$

where

$$
T(m ; p, M)=\frac{\pi}{p}\left\{1+\frac{p-1}{1+\exp (m-M / 2)}\right\} .
$$

where $p$ is a constant determined by the phase stability obtained after the phase- 1 phase stabilization. Note that Eq. (4) is a monotonically decreasing sigmoid function with the maximum limit of $\pi$ and the minimum limit of $\pi / p$, and we set $p$ to make $\pi / p$ to be the phase noise level. In our particular case, 
$p=12 . M$ is the total iteration number of iteration, and in our particular case, $M=7$. Note that Eq. (4) is a sigmoid function. And hence, In this condition, $T(m ; p, M)$ keeps large for the first iterations, rapidly decreases at around the middle of the iteration, and becomes relatively constant for the late steps of the iterations. Hence this algorithm estimates the phase error caused by the system instability and the bulk eye motion more accurately than our previous algorithm, and improves the image quality of Doppler tomography and OCA.

After applying the phase-2 stabilization, we applied a 3-D Kasai auto-correlation filter ${ }^{33,34}$ to the Doppler signal to further improve the Doppler sensitivity as described in Section 2.3 of Ref. 32. In this particular study, we have further optimized the kernel size of this filter in comparison to that of Ref. 32. The kernel sizes utilized in this study were 4 pixels (depth) $\times 4$ pixels (horizontal) $\times 1$ pixel (vertical) for the bi-directional scanning mode, and 4 pixels $($ depth $) \times 1$ pixel (horizontal) $\times 4$ pixels (vertical) for the high-sensitive scanning mode. This kernel sizes correspond to the physical size $19.0 \mu \mathrm{m}$ (depth) $\times 38.8$ $\mu \mathrm{m}$ (horizontal) $\times 30.0 \mu \mathrm{m}$ (vertical) for the bi-directional mode and $19.0 \mu \mathrm{m}$ (depth) $\times 30.0 \mu \mathrm{m}$ (horizontal) $\times 38.8 \mu \mathrm{m}$ (vertical) for the high-sensitive mode. 